\title{
Endometrial Curettage When and for Who
}

\author{
Aslıhan Duman ${ }^{1}$, Ferah Tuncel Daloğlu ${ }^{2}$, Yeliz Arman Karakaya ${ }^{3}$, Aysun Aras ${ }^{4}$ \\ ${ }^{1}$ Giresun University Faculty of Medicine,Department of Medical Pathology, Giresun, Turkey \\ ${ }^{2}$ MersinToros State Hospital, Pathology Specialist, Mersin, Turkey \\ ${ }^{3}$ Isparta State Hospital, Pathology Specialist, Isparta, Turkey \\ ${ }^{4}$ Ağrı Public Health Directorate, Public Health Specialist, Ağrı, Turkey
}

Email address:

ferahdaloglu@hotmail.com (F. T. Daloğlu),yelizkaraya20@gmail.com (Y. A. Karakaya), draysunaras@windowslive.com (A. Aras)

\section{To cite this article:}

Aslıhan Duman, Ferah Tuncel Daloğlu, Yeliz Arman Karakaya, Aysun Aras. Endometrial Curettage When and for Who. Journal of Cancer Treatment and Research. Vol. 3, No. 2, 2015, pp. 17-21. doi: 10.11648/j.jctr.20150302.11

\begin{abstract}
In this study, the histopathological diagnoses pertaining to curettage material and the clinical necessity and results of endometrial curettagein age groups of patients in our region who had curettage due to abnormal uterine bleeding were studied. Thehistopathological diagnoses of patients with the above-mentioned criteria were scanned, excluding those related to pregnancy. 409 cases were re-examined and evaluated by a group consisting of three pathologists. Diagnoses were classified into 10 different groups. According to this classification system, out of the total number of cases, 100 were defined as endometrial polyp (24.4\%), 72 cases as secretory endometrium (17.6\%), 59 cases as proliferative endometrium (14.4\%), 51 cases as stromal/glandular degeneration (12.5\%), 32 cases as irregular proliferation (7.8\%), 26 cases as insufficient (6.4\%), 22 cases as chronic endometritis (5.6\%), 18 cases as decidualization (4.4\%), 17 cases as endometrial hyperplasia (complex atypical)/ adenocarcinoma (4.2\%), and 12 cases as others (inactive, atrophic, basal and menstrual endometrium) (2.9\%). The average age of the 409 cases was $44.8 \pm 7.8$. Upon evaluation of curettage due to abnormal uterine bleeding in the approximate 3 year period in our region, and taking into consideration the age distribution, it was observed that the clinical pre-diagnoses of patients especially with endometrial polyps and precancerous lesions were coherent with the corresponding histopathological diagnoses.
\end{abstract}

Keywords: Endometrium, Curettage, Histopathological Diagnosis, Age

\section{Introduction}

Abnormal uterine bleeding (AUB) is the most common reason for admissions to gynecology clinics after vaginal infections. Chronic endometritis, endometrial polyp, endometrial hyperplasia andadenocarcinoma are usually considered to be among the reasons underlying AUB (3).

Malignant or premalignant endometrial lesions are most commonly determined by AUB (3). Endometrial sampling is important for accurate histopathological diagnosis. Starting from delivery to pathology laboratory, the endometrial sample passes through a series of diagnostic processes.Both macroscopic and microscopic descriptionsare valuable for histopathological diagnosis (1-13). The descriptions are as follows:

Insufficient term: Occasionally the material may only consist of blood and mucous or just fragments of endometrium surface epithelium. Curettage of menopausal or post-menopausal endometrium may be void of any tissues.
Decidualization term: Indicates transition of stromal cells from proliferative stage to cells withunclear cytoplasm boundaries, large oval and round vesicle nucleus, with eosinophilic or clear cytoplasm.

When this transition is less evident, it is referred to as predecidual stage and is an indication of the late secretory period of normal menstruation cycle.

Secretory endometrium: It has been stated that the most appropriate time for verification of ovulation is on the $22^{\text {nd }}$ day of cycle or afterwards. The first reliable histological change specific to ovulation is observed at the $17^{\text {th }}$ day of the cycle.

These are well developed sub-nuclear vacuolization and nuclear palisading in all cells and glands. On $18^{\text {th }}$ day of cycle supra-nuclear vacuolization begins to be seen. In days 19 and 20, supra-nuclear cytoplasmic product is excreted to the gland lumen through apocrine secretion. After $20^{\text {th }}$ day of cycle, stroma evaluation is done instead of gland evaluation.

These changes are edema, folding of the spiral arterioles 
and stroma predecidualization.

Maximal stromal edema occurs on $22^{\text {nd }}$ day.

Endothelial proliferation causes folding in the endometrium arterial system. In histological sections vascular groups are visible on the upper functional layer.

Stromal predecidualization begins on days 22-23. On the functional layer, it is on the spiral arteriole and capillary surroundings. Predecidual transformation of stromal cells below superficial epithelium occurs on $25^{\text {th }}$ day. The joint decline of estrogen and progesterone on day 25 of the cycle fails to maintain the integrity of the lysosomal enzymes. As a result, lysosomal enzymes are released inside the cells and in the cavity between them.

Proliferative endometrium:Vascular endothelial cells, together with glandular and stromal cells are characterized by proliferation. This proliferation results in increased mucosa of theuterus. It consists of numerous mitoses. The normal proliferative glands of endometrium consist of oval-roundshaped glands that exhibit changes in diameter and fold according to early, middle or late stage and include nuclear pseudo-stratification and mitosis. As glands in early stage of proliferation advance to the middle and late stages, they acquire amore twisted and voluminous shape compared with straight tubules. In addition, the epithelial cells of surface epithelium and especially gland lumens gain cilia and microvilli. Lymphoid aggregates reminiscent of follicles may be seen in endometrial stroma, especially during the proliferative stage.

Endometritis:Endometrial inflammation is a pathology generally belonging to the fertility period, which can appear in infectious or non-infectious cases. Hence, it is actually a histopathological entity. Clinical correlation is not always possible. It is possible to classify edometrium inflammations as specific or non-specific. Non-specific endometritis are more commonly seen. Since some inflammation elements may be present in the endometrium stroma under normal conditions, the inflammatory cells of the endometrium must always be evaluated as a component of endometritis. Especially the granulocites of secretion phase and polymorphonuclear leukocytes of the menstrual phase must not be accepted as symptom of inflammation. In acute inflammation there is an infiltration consisting of polymorphonuclear leukocytes, which collect in gland lumens and form micro-abcesses and are also present in surface epithelium. The presence of PNL alone is not an evidence for inflammation as they are also present in the menstrual phase. In chronic endometritis there is an inflammation with a predominancy of plasma cells, lymphocytes and macrophages. Small amounts of polymorphonuclear leukocytes and eosinophils may be present.

Endometrial polyps:Since these polyps are seen as fragments in curettage material, it is sometimes difficult to distinguish them from normal endometrium and endometrial hyperplasia. Polyps appear as larger particles compared with normal endometrium, and are round-shaped particles surrounded by surface epithelium. The stroma is usually dense and includes thick-walled vessel sections. The glands do not have the uniform orientation of normal endometrium and have variable shapes and sizes. At times they exhibit the normal typical secretory or proliferative activity, in which case they are named as "functional polyps".

In pathology reports, polyps are classified as

- adenomatous

- adenofibromatous

- adenofibromyomatous

- adenomyomatous

according to the appearance and ratio of glandular and stromal elements.

The polyps located at the lower segment and proximal endocervix sometimes have both endometrial and endocervical type epithelium on the surface or glands. These are called "endocervical-endometrial polyps".

Endometrial Hyperplasia:The uterus is usually large. The curettage materialfills 3 or more cassettes. Hyperplasia is usually a diffuse abnormality; it covers most of the endometrium and is often polypoid-occurring. Gland and stroma together may exhibit increase. In some cases, especially atypical hyperplasia, it can be a localized lesion. The atypical diagnosis is primarily based on nucleus features; most of them change from oval to round shape, become enlarged, irregularly-shaped, and have non-uniform membrane with 2-4 lines true stratification. There is irregular stratification and loss of polarity. There is variable mitotic activity. Chromatin forms clusters along the nuclear membrane and lead to the vesicular appearance characteristic of endometrial atypia. The nucleolus may be apparent. Since the atypia in hyperplasia is usually focal, the cytoplasmic eosinophilia may be the first symptom to warn the pathologist.

Endometrioid adenocarcinoma:Often occurs on the basis of endometrial hyperplasia. They are defined as endometrial carcinoma due to their resemblance to the glandular structure of normal endometrium.

Below items must be taken into account in differentiating endometrioid adenocarcinoma diagnosis from atypical hyperplasia

- Complex and cribriform pattern

- $\quad$ Epithelial bridge

- $\quad$ Bizzare-shaped glands

- Mitosis (sometimes atypical)

- Nucleus abnormalities: nucleomegaly, chromatin transparency, evident nucleolus

- Presence of luminal neutrophile

Moreover, evident desmoplastic stroma separating glands and gland masses can be observed in endometrioid adenocarcinoma.

Irregular proliferation: Developes as a result of prolonged, unfulfilled estrogen effect (similar to chronic anovulatory cycles). It is not a real premalign lesion.

Stromal/glandular degeneration:A non-specific symptom. It can also be observed in bleedings accompanying menstrual endometrium and different organic diseases. Endometrium areas that have remained healthy must be carefully examined 
for specific pathological findings (such as polyps, hyperplasia and malignancy. Nuclear debris in stroma (apoptotic), fibrin thrombi, stromal collapse, stromal cell aggregates, papillary syncytial changes in glands, glandular impingement, nuclear debris in basal cytoplasm of glandular cells (apoptotic), storage of hemosiderin pigment, fibrosis and hyalinization occur.

Menstrual endometrium:Occurs on day 28 of cycle following glandular and stromal degeneration. Apopotic bodies become evident in stroma and glands. Clustered stromal cell groups (stromal blue balls) gather together and are seen in the degenerated stroma.

Following degeneration, glandular cells take a form similar to micropapillarystructure, this is defined as a regenerative change and called papillary syncytial metaplasia.

Inactive endometrium:An endometrium with sufficient thickness but failing to exhibit morphological characteristics toward hormonal stimuli, such as active proliferative or secretive activity. These morphological changes can be found in most menopausal and post-menopausal women with decreased ovarian hormones, which are not sufficient enough to start endometrial proliferation. It recalls proliferative endometrium, however glands are usually parallel to surface epithelium, in contrast to perpendicular orientation in proliferative endometrium. They have pseudostratified nucleus without mitosis, and ciliated cells are rarely observable. The stroma has dense characteristic regardless of basal and fuctional distinction.

Atrophic endometrium:Consists of small atrophic tubulles and cystic dilated glands scattered in fibrous stroma in wide spaces, usually exhibiting no proliferative activity in postmenopausal stage (13).

\section{Findings}

In this study, retroactive analysis of histopathological diagnoses of curettage materials obtained in 2011-2013 as a result of AUB was performed. The histopathological diagnoses were scanned, excluding those related to pregnancy, and 409 cases were obtained. The cases were reexamined and evaluated by 3 pathologists. These were then gathered under 10 different diagnosis headings in histopathological terms. According to this classification system, out of the total number of cases, 100 were defined as endometrial polyp (24.4\%), 72 cases as secretory endometrium (17.6\%), 59 cases as proliferative endometrium (14.4\%), 51 cases as stromal/glandular degeneration (12.5\%), 32 cases as irregular proliferation $(7.8 \%), 26$ cases as insufficient (6.4\%), 22 cases as chronic endometritis (5.6\%), 18 cases as decidualization (4.4\%), 17 cases as endometrial hyperplasia (complex atypical)/ adenocarcinoma (4.2\%), and 12 cases as others (inactive, atrophic, basal and menstrual endometrium) (2.9\%), (Graph 1). The ages of 409 cases were analyzed, with a minimum value of 20 and maximum value of 70 , and average age of $44.8 \pm 7.8$. The average age of each diagnostic group was calculated separately (Table 1). The difference between diagnostic groups and average age was found to be significant. Single direction variance analysis was used as $\mathrm{P}=0.01$ test. The difference originated from the averages of groups 2, 6 and 9 .

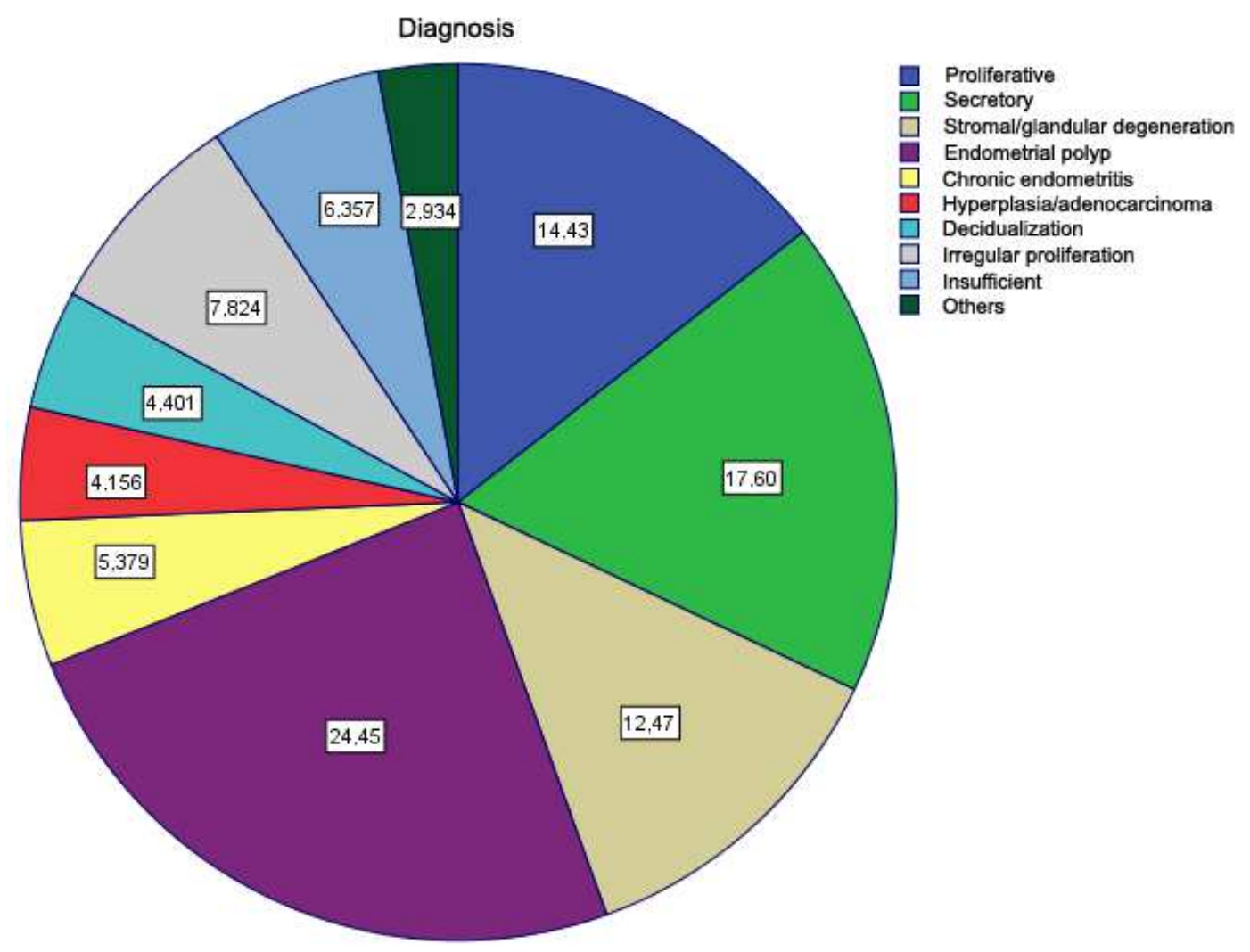

Graph 1. Distribution of patients according to their diagnosis. 
Table 1. Age averages according to diagnostic groups and analysis of diagnostic group distributions.

\begin{tabular}{lllll}
\hline DIAGNOSIS & Average \pm SS & Min.-Max. Value & Number & \% \\
\hline 1.Proliferative & $43.69 \pm 6.7$ & $23.0-57.0$ & 59 & 14.4 \\
2.Secretory & $43.09 \pm 7.5$ & $20.0-59.0$ & 72 & 17.6 \\
3.Stromal/GlandularDegeneration & $43.68 \pm 8.2$ & $22.0-66,0$ & 51 & 12.5 \\
4.Endometrial Polyp & $46.20 \pm 7.6$ & $22.0-63.0$ & 100 & 24.4 \\
5.Chronic Endometritis & $44.59 \pm 6.8$ & $33.0-52.0$ & 22 & 5.4 \\
6.Hyperplasia/Adenocarcinoma & $49.17 \pm 8.2$ & $34.0-63.0$ & 17 & 4.2 \\
7.Decidualization & $45.44 \pm 7.6$ & $27.0-56.0$ & 32 & 4.4 \\
8.Irregular Proliferation & $43.78 \pm 6.1$ & $33.0-53.0$ & 26 & 7.8 \\
9.Insufficient & $48.46 \pm 10.9$ & $26.0-70.0$ & 12 & 6.4 \\
10.Others & $42.25 \pm 9.7$ & $27.0-530$ & & 2.9 \\
TOTAL & & & 409 & 100 \\
\hline
\end{tabular}

\section{Discussion}

In all age groups, especially women in reproductive age,AUB constitutes the second place among the causes of visits to the gynecology clinic, after vaginal infections (1-6). Most of the endometrial samples are obtained from women in pre-, peri- or post-menopuasal stage $(3,6)$. The traditional method is cervical dilatation and curettage. It is believed that this method is therapeutic and has a good sample forming property for histopathological evaluation (13).

Saraswathi et al. (1) have emphasized that abortion in young patients increases the probability of AUB, and that this must be investigated in the reproductive period excluding pregnancy complications.

In our study, the histopathological diagnoses were scanned, excluding those pertaining to pregnancy.

In the same study, chronic endometritis was detected in some patients, and in one patient epithelioid granuloma supporting tuberclosis was detected (1).

In our study, 22 patients out of 409 cases had chroic endometritis.

Since these are the most common reasons for anemia in young women in pre-menopausal stage and due to the presence of suspected malignancy in post-menopausal stage, the evaluation, diagnosis and treatment of AUB and postmenopausal bleeding cases are important from the gynecological point of view (3).

In the study performed by Çakmak et al. (3) on 400 cases, they have come to believe in the application of endometrial sampling on selected cases rather than applying it as routine procedure in menometroragia cases, yet with the possibility of having a more liberal application in post-menopausal bleeding cases, and the unnecessity of its routine application prior to operation in planned hysterectomy due to myoma, but the importance of its application for endometrial evaluation in cases where cervical polyp has been detected.

In our study, however, $24.4 \%$ of the cases with AUB complaint were diagnosed as endometrial polyp, and the average age of this patient group being 46 shows that endometrial sampling is indeed effective in this diagnostic group.

Endometrial polyp is a benign disease affecting $25 \%$ of women, and has been detected in $13-50 \%$ of women with AUB complaints (2). In a retrospective study carried out by Lasmar et al. (2) on hysterectomy patients, $14.8 \%$ of endometrial polyps with diameters larger than $15 \mathrm{~mm}$ hyperplasia were found to have hyperplasia, and therefore concluded that the increase in polyp diameter increases the possibility of hyperplasia development.

All 409 cases in our study were endometrial curettage material and 100 cases were endometrial polyp.

In a research carried out by Sweet et al. (6) on patients defined as AUB for various reasons, they have concluded that the most effective treatment of pre-menopausal women is hysterectomy.

Oğuz et al. (12) have detected that in 12 out of 58 hyperplasia patients, atypic endometrial hyperplasia has developed into adenocarcinoma.

In compliance with literature, since the possibility of complex atypic hyperplasia developing into adenocarcinoma is high, we have formed one of the diagnostic groups as hyperplasia/adenocarcinoma.

In conclusion, in the retrospective analysis of 409 cases and evaluation in light of age range, the highest age (70) was detected in the insufficient category, the secong highest age (66) in stromal/glandular degeneration category, and the third highest age (63) in endometrial hyperplasia/adenocarcinoma and endometrial polyp categories. According to these findings, due to the average age being $44.8 \pm 7.8$, it has been found that the clinical/histopathological diagnoses of endometrial curettage carried out on patients in reproductive age are in agreement with one another.

\section{References}

[1] Doraiswami S., Johnson T., Rao S., Rajkumar A., Vijayaraghavan J., Panicker VK. Study of Endometrial Pathology in Abnormal Uterine Bleeding.J Obstet Gynaecol India. 2011; 61(4):426-30.

[2] Lasmar BP., Lasmar RB. Endometrial polyp size and polyp hyperplasia.Int J Gynaecol Obstet. 2013 ;123(3):236-9

[3] Çakmak B., Karataş A., TuranG. Endometrial Örnekleme Sonuçlarımız: 400 Olgunun Analizi.Selçuk Tıp Derg. 2012; 28(3):163-166 
[4] Goertzen J., Hutten-Czapski P. The occasional endometrial biopsy.Can J Rural Med. 2010; 15(3):113-7

[5] Carvalho FM, Aguiar FN, Tomioka R, de Oliveira RM, Frantz $\mathrm{N}$, Ueno J. Functional endometrial polyps in infertile asymptomatic patients: a possible evolution of vascular changes secondary to endometritis.Eur J Obstet Gynecol Reprod Biol. 2013; 170(1):152-6.

[6] Sweet MG, Schmidt-Dalton TA, Weiss PM, Madsen KP. Evaluation and Management of Abnormal Uterine Bleeding in Premenopausal Women. Am Fam Physician. 2012 ; 85(1): 3543.

[7] Dascanio JJ, McCuePM, Endometrial Biopsy. John Wiley \& Sons. Published Online: 27 JUN 2014 DOI: 10.1002/9781118904398.ch19

[8] Saadia A, Mubarik A, Zubair A, Jamal S, Zafar A.Diagnostic accuracy of endometrial curettage in endometrial pathology.J Ayub Med Coll Abbottabad. 2011 ; 23(1):129-31.
[9] Silverberg SG.The Endometrium Pathologic Principles and Pitfalls. Arch Pathol Lab Med. 2007; 131: 372-82

[10] McCluggage WG. My approach to the interpretation of endometrial biopsies and curettings. J Clin Pathol. $2006 ; 59(8): 801-12$

[11] İvit H., Köksal A., Çukurova K., Keklik A., Yıldız A., Yetimalar H., Sapmaz L. Postmenopozal kanamalı olguların endobrush endometrial örnekleme yöntemiyle değerlendirilmesi. SSK İzmir Eğitim Hastanesi Tıp Dergisi. 2004, 10 (1): 15-19.

[12] Endometrial hiperplazi tanısı verilen hastaların prognozlarının retrospektif olarak değerlendirilmesi Oğuz S., L. Gözeri L.İ., Akdoğan E.,K., Saraç G., Akyağc1 A.

[13] Blaustein's Pathology of the Female Genital Tract Kurman, Robert J., Hedrick Ellenson, Lora, Ronnett, Brigitte M. (Eds.)6th ed. 2011,page 305- 452. 